\title{
Relative Timing of Variability of Blazars at X-Ray and Lower Frequencies
}

\author{
Alan P. Marscher*, Svetlana G. Jorstad* ${ }^{* \dagger}$, Margo F. Aller**, Ian McHardy, Thomas \\ J. Balonek ${ }^{\S}$, Harri Teräsranta ${ }^{\text {II }}$ and Gino Tosti ${ }^{\|}$ \\ *Institute for Astrophysical Research, Boston University, 725 Commonwealth Ave., Boston, MA 02215 \\ ${ }^{\dagger}$ Sobolev Astronomical Institute, St. Petersburg State University, Universitetskij pr. 28, 198504 St. Petersburg, \\ Russia \\ ${ }^{* *}$ Astronomy Department, University of Michigan, 830 Dennison, 501 East University Ave., Ann Arbor, MI \\ 48109-1090 \\ ${ }^{\ddagger}$ Department of Physics and Astronomy, University of Southampton, Highfield, Southampton, SO17 1BJ, United \\ Kingdom \\ ${ }^{\S}$ Department of Physics and Astronomy, Colgate University, 13 Oak Dr., Hamilton, NY 13346-1398 \\ IMetsähovi Radio Observatory, Helsinki University of Technology, Metsähovintie 114, Kylmala, Finland-02540 \\ "Dipartmento di Fisica, Università di Perugia, Perugia, Italy
}

\begin{abstract}
The rich X-ray light curves of blazars obtained with RXTE allow meaningful correlation analyses with longer wavelengths. This reveals strong connections of variations across the electromagnetic spectrum. In 3C 279, PKS 1510-089, and BL Lac, the characteristics of the X-ray variability change along with the projected direction of the compact jet. Outbursts in the radio, IR, or optical often precede flares at high energies. A period of pronounced variability in BL Lac in late 2000 occurs at both optical and X-ray frequencies, with the X-ray spectral index steepening. A superluminal radio knot is ejected during this event. The implication of our monitoring results is that the IR to X-ray (as well as $\gamma$-ray) emission is cospatial with the compact radio jet, most likely occurring in the superluminal knots. In the radio galaxy $3 \mathrm{C} 120$, in which the X-rays probably come mainly from a hot accretion-disk corona, the appearance of superluminal radio knots follows (by 4 weeks) dips in the X-ray emission, as in microquasars but on longer timescales. The delay implies that the core of the radio jet, as seen in $\mathrm{mm}$-wave VLBA images, lies at least $0.4 \mathrm{pc}$ from the central engine, consistent with models in which the jet flow accelerates far from the black hole. The quasar 3C 273 may be an interesting hybrid case in which contributions to the X-ray emission may come from both the jet and corona. The power spectral density has a low-frequency break that, in analogy with black-hole binary systems, implies a mass of the central black hole of $3-6 \times 10^{8} M_{\odot}$, similar to that obtained by reverberation mapping of emission-line variability.
\end{abstract}

\section{INTRODUCTION}

The superb monitoring capability of RXTE allows the study of X-ray variability in an effort to probe the physical conditions of blazars. Indeed, RXTE observations of radio-loud quasars have shown that the $\mathrm{X}$-ray emission is much more interesting than had been realized previously. The flux is highly variable on timescales of days or weeks in all the blazars that have been monitored, and in some objects X-ray variations are highly correlated with those at lower frequencies (see Figs. 1-5). This provides an excellent opportunity to use variability to probe both the general characteristics and the details of the relativistic jet flows and nonthermal X-ray emission processes in blazars.

One of the most important goals of multiwavelength timing studies of blazars is to locate the site of the X-ray emission (the accretion disk? the radio jet? somewhere between the two?) and its relation to the gross properties of the relativistic jet such as its direction and bulk Lorentz factor. After this fundamental information is established, one can use the observations to probe the nature of the particle acceleration and energy losses, the formation and propagation of shock waves, the changing geometry and strength of the magnetic field, and the relation between the supermassive black hole/accretion disk and the relativistic jet.

Here we report on a long-term multiwaveband monitoring program that follows the variations in emission of selected objects with closely-spaced observations over time spans as long as 7 years. We supplement multiwavelength light curves with frequent VLBA images that provide direct information on the behavior of the jets on parsec scales. Based on these data, we conclude that the IR, 
optical, X-ray, and $\gamma$-ray emission is cospatial with the most compact sections of the radio jet as observed with the VLBA at millimeter wavelengths.

\section{THEORETICAL EXPECTATIONS}

Most theorists consider the synchrotron self-Compton (SSC) mechanism as the heavily favored candidate for the dominant $\mathrm{X}$-ray emission process in non-TeV blazars. If this is the case, then we expect strong correlations between the lower frequency (synchrotron) variations and those in the X-ray. If the source is uniform and if the timescale of the changes in the number of radiating particles and magnitude of the magnetic field is longer than the light-travel time across the emitting region, then flares should be simultaneous at all wavelengths. However, the timescales are often quite short and uniformsource models rarely work well.

As is discussed in Sokolov and Marscher (these proceedings), the general, probably more realistic, case is quite interesting. The geometry of the particle acceleration region and internal light-travel delays are important when the timescales are short. Frequency stratification of the synchrotron emission occurs when the electrons are energized at a front (e.g., a shock front) and then advect away at a speed close to $c$ as they lose energy via radiative losses [1]. This causes the optical emission to emanate from the region immediately behind the shock front, with the infrared emission arising from a somewhat larger region behind the front and the radio from an even more extended volume. The synchrotron variations will then have time delays, with those at higher frequencies occurring earlier.

The relative timing of the SSC X-ray emission is not as simple to estimate. As pointed out by [2], the X-ray SSC emission comes from a wide spectrum of seed photons, with rest-frame frequencies $\sim 10^{10}-10^{15} \mathrm{~Hz}$, which are scattered by electrons with energies $E \sim 10^{1}-10^{5} m c^{2}$. In fact, the contribution is roughly the same for each decade of seed-photon frequency. If there is a frequency dependence of the spatial distribution of seed photons, as in the case of shock-front particle acceleration, one needs to carry out numerical computations to determine the theoretical X-ray light curve. Furthermore, there is an internal light-travel delay for the seed photons to reach the electrons that scatter them to X-ray energies. The result is that "reverse" time delays should occur, with the $\mathrm{X}$-ray lagging behind the optical/IR variations.

In radio-loud objects that are not blazars in the optical-such as the radio galaxy 3C 120 and the quasar 3C 273 in our study - it is not clear whether the jet X-ray emission is stronger or weaker than that from electron scattering in a hot corona above and below the accre- tion disk, as seen in lower luminosity active galactic nuclei (AGN). If the latter dominates, then a low-frequency break in the power spectral density of the X-ray light curve provides an estimate of the mass of the black hole (see McHardy, these proceedings).

\section{REVERSE TIME LAGS IN THE BLAZARS PKS 1510-089 AND 3C 279}

A prime example of reverse time delay occurs in the quasar PKS 1510-089 (see Fig. 2, upper left inset). The radio and X-ray light curves in 1997, 1998, and 2003 are well correlated, with the radio variations leading the $\mathrm{X}$ ray by 9-17 days as indicated by the peak in the discrete cross-correlation function (DCCF). In 1999-2001, the correlation is generally weaker and the lag is in the opposite sense, while in 2002 the correlation is strong but at zero lag. During the period of weakest correlation, the direction of the jet is considerably farther to the south, while the intervals of strongest correlation-with reverse time lags - correspond to the northernmost direction of the jet (see the upper right inset to Fig. 2). As we continue to observe this object, we should be able to determine whether this relationship between jet direction and the nature of the X-ray/radio correlation occurs consistently.

The reverse time lag can only be explained if the $\mathrm{X}$-rays are produced in the radio jet-in the core or a bit downstream-rather than closer to the central engine. Furthermore, it suggests that the flares are lighttravel limited, since otherwise the SSC internal lighttravel time delay discussed in the previous section does not apply.

In 3C 279, the overall light curve (Fig. 3) suggests an R-band/X-ray correlation. This is verified by the DCCF (Fig. 4), which indicates a strong (peak in DCCF $0.77 \pm$ $0.19,>99.999 \%$ confidence) R-band/X-ray correlation at a lag of $16 \pm 3$ days, with the optical leading the $\mathrm{X}$-ray. Since the seed photons that are scattered to Xray energies could be predominantly from IR or optical wavelengths, the cause of this reverse lag could be either SSC light-travel delays or frequency stratification, or both.

As shown in Fig. 5 (middle panel), the apparent direction $\Theta$ of the milliarcsecond-scale jet of 3C 279 swung by over $30^{\circ}$ during our RXTE monitoring. Furthermore, the apparent speed of the radio knots increased from $5 c$ in 1996 to $13 c$ in 1998 to $26 \pm 3 c$ in 2001 , when the optical and X-ray activity was most violent. The apparent speed then decreased precipitously to $\sim 7 c$ in 2002 while the $\mathrm{X}$ ray flux and level of variability declined monotonically to the lowest levels seen during our program. The continuation of our monitoring program (approved through March 2005) should allow us to determine whether this 




FIGURE 1. X-ray and radio light curves of PKS 1510-089. Arrows show times (horizontal lines: uncertainties) of known ejections of superluminal radio knots; some ejections may have been missed by the more limited time coverage prior to 1998.3 owing to the extremely rapid motions (as high as $45 c$ for $H_{0}=65$ ). VLBA data that would indicate ejections after 2002.5 are still being analyzed. In the lower panel, the filled circles, open circles, and small triangles correspond to 14.5, 22, and 37 $\mathrm{GHz}$, respectively.

apparent correlation between the X-ray behavior and the jet direction and apparent speed is real (i.e., repeats) or just coincidental. Furthermore, relating the emission properties to projected direction and apparent speed can lead to a determination separately of the Lorentz factor and angle to the line of sight of the jet.

Statistical analysis of the relative timing of high levels of $\gamma$-ray flux and epochs of ejection of superluminal radio knots of blazars indicates that the $\gamma$-ray variations also lag behind those observed at high radio frequencies [3]. It therefore appears that the high-energy emission is co-spatial with the radio emitting portion of the compact jet. This conclusion is surprising in light of the short timescales of variability at high energies. However, the relativistic beaming factors of $\gamma$-ray bright blazars is very high [4], hence the timescales are greatly foreshortened in the observer's frame. In addition, the narrow opening angles of the jets allows small dimensions of compressed emission regions even if those regions lie parsecs downstream of the central engine.

If the high-energy emission comes from the superluminal radio knots, then there should be a correspondence between epochs of superluminal ejections and Xray flares perhaps with a time delay. While many X-rays flares are accompanied by the appearance of new super-

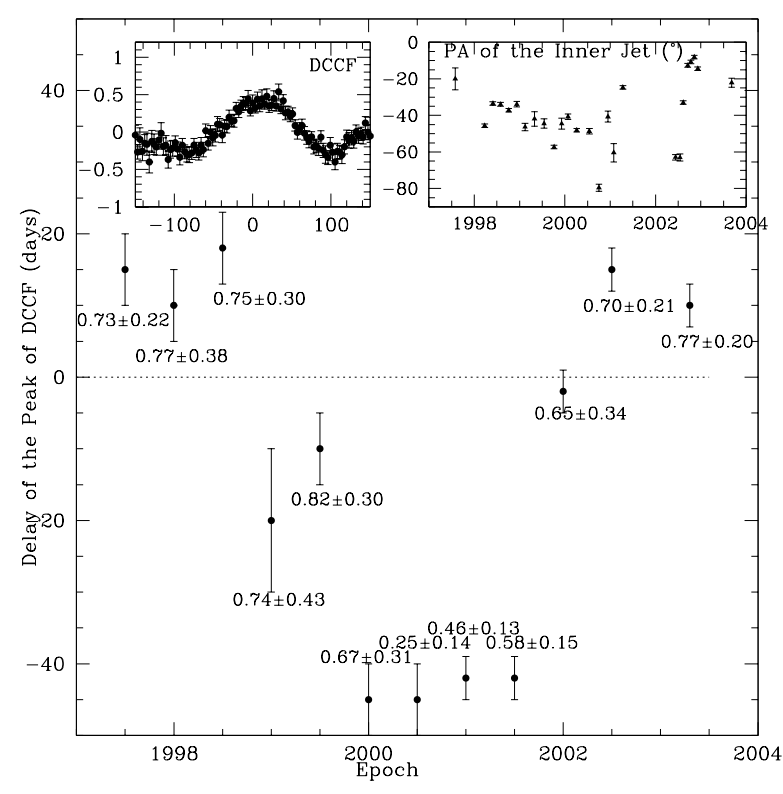

FIGURE 2. Delay of peak in the discrete cross-correlation function (DCCF) of the $14.5 \mathrm{GHz}$ and X-ray light curves for each half year of observation; data from 6 months before to 6 months after each point are included in the DCCF. Positive values correspond to radio leading. The value of the DCCF peak is given, with those above $\sim 0.5$ corresponding to high levels of significance. Left inset: DCCF for all the data. Right inset: Direction of the pc-scale jet as a function of time.

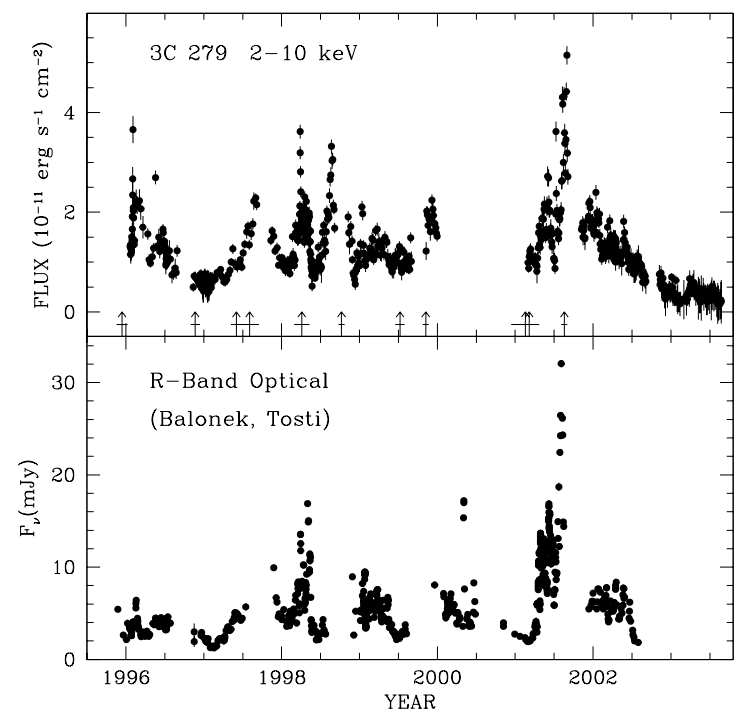

FIGURE 3. RXTE and optical light curves of 3C 279. Arrows show times of known ejections of superluminal radio knots; any ejections in mid-1996 may have been missed owing to a dearth of VLBA observations during this time period, and no information is yet available for ejections after 2002.5. (Ejections during 2000 when we did not observe with RXTE are not shown in the plot.) 


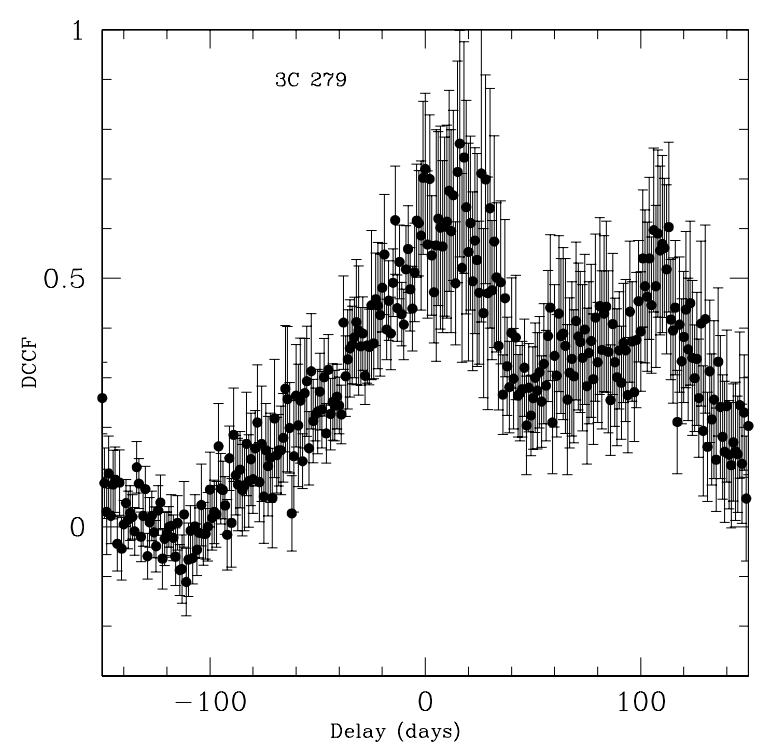

FIGURE 4. Discrete cross-correlation function between the entire X-ray and optical light curves of 3C 279. Positive values correspond to the optical leading the $\mathrm{X}$-ray.

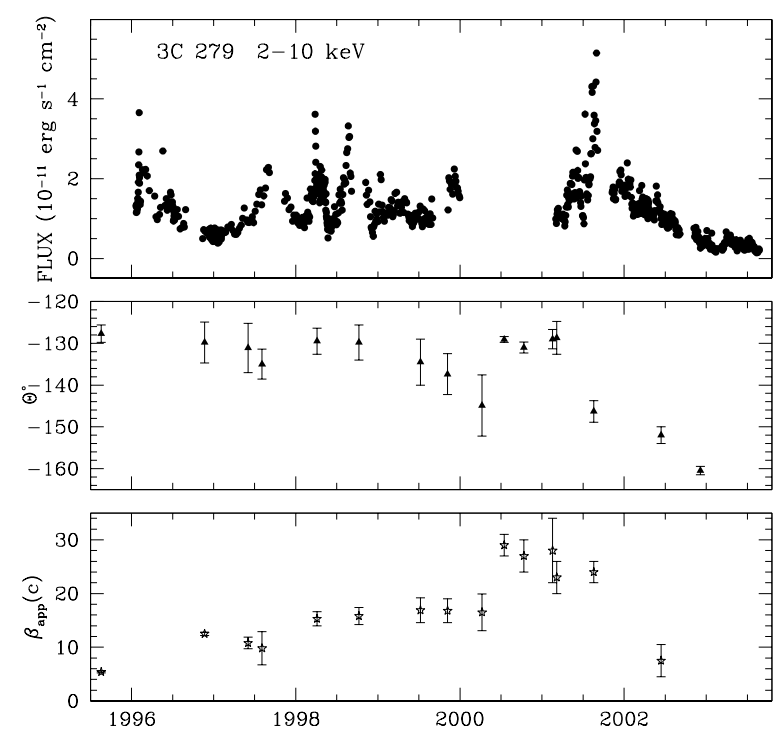

FIGURE 5. Repeat of the X-ray light curve (top), angle of jet close to the core (middle), and apparent speed of superluminal knots (for $H_{0}=65$ ) vs. date of extrapolated coincidence with the core (bottom).

luminal knots, the correspondence is not one-to-one. The analysis is complicated by the large numbers of both major and minor flares and superluminal ejections per year in these two blazars. The latter causes "crowding" of bright spots in the millimeter-wave images of 3C 279 , so that it is possible to miss some ejections and to assign an ejection time to a feature that actually represents a bifurcation of what initially was a single knot. Finally, the radio flux of some knots increases with time out to a few tenths of a milliarcsecond from the core; if the X-ray flux also increases during this stage, then the peak of the $\mathrm{X}$ ray flare will be displaced by months from the time when the knot was coincident with the core of the jet. We are in the midst of a more detailed analysis to sort out these complexities.

If the flares are light-travel-time limited, then it is a challenge to explain why the profiles are usually sharply peaked. Such flares should have $\sim$ flat tops unless the decay time equals light-travel time across the emitting region. It therefore seems that there is an ever-present strong decay process that is overcome for some period by an even stronger rise mechanism. One possibility is that the rise is caused by an increase in the Doppler factor either by bending of the trajectory of the emission region into the line of sight or by an acceleration of the jet flow with distance from the central engine. The latter can occur out to parsec scales in the model of Vlahakis and Königl [5], in which magnetic forces both collimate and accelerate a relativistic jet.

\section{BL LAC IN 1999-2001}

Our monitoring of BL Lac has been more limited, covering a two-year period with a 5-month gap. Fig. 6 shows the X-ray and optical light curves along with the times of ejection of superluminal radio knots. X-ray and optical light curves in year 2000, including some of the data shown in Fig. 6, have been published by [6].

Unfortunately, the gap in our RXTE coverage caused us to miss a prolonged optical outburst, which corresponded to a radio flare and superluminal ejection. But we did observe a several month period of violent variability at both X-ray and optical frequencies in late 2000. A new superluminal radio knot appeared during the interval of heightened activity. During this period, the X-ray spectrum was steeper than usual, which suggests that the enhanced X-ray fluxes may correspond to synchrotron radiation that rises above the inverse Compton emission level of $\sim 1 \times 10^{-11} \mathrm{erg} \mathrm{cm}^{-2} \mathrm{~s}^{-1}(2-10 \mathrm{keV})$.

The multiwaveband data suggest that there is a close correspondence between X-ray, optical, and radio activity in BL Lac. It is possible that the variability characteristics change as the direction of the jet swings [7], as appears to be the case in PKS 1510-089 and 3C 279. However, a longer data train would be necessary to establish whether this is the case. 


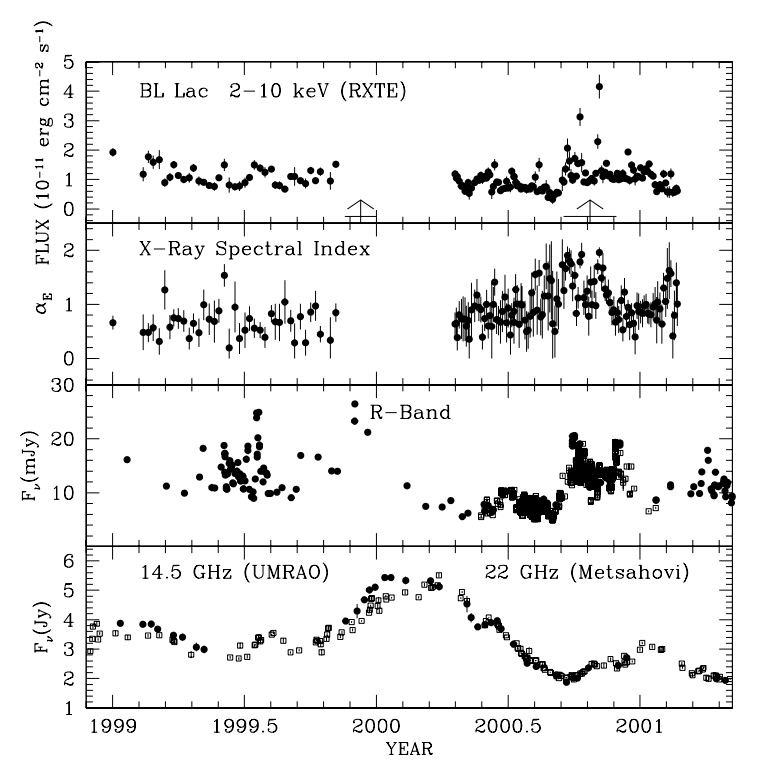

FIGURE 6. RXTE and optical light curves of BL Lac. Arrows show times of ejections of superluminal radio knots. The Villata et al. data are from [8]. In the bottom panel, filled circles correspond to $22 \mathrm{GHz}$, while open squares denote $14.5 \mathrm{GHz}$ data.

\section{THE RADIO GALAXY 3C 120: A MACRO MICROQUASAR}

Hybrid objects can provide extremely important checks on the unification of accreting systems with different black-hole masses. The radio galaxy 3C 120 is a case in point. It is blazar-like in the radio [9] but Seyfert-like in the optical and X-ray [10]. Given the similarities between Seyferts and black-hole binary systems (see McHardy's paper in these proceedings), and the common characteristic of relativistic jets in 3C 120 and microquasars [11], we decided to search for a phenomenon that had previously been detected only in microquasars: dips in X-ray flux followed by the appearance of superluminal radio knots in the jet.

As we reported in [12], 3C 120 cooperated. As shown in Fig. 7, each superluminal ejection between 1997.0 and 2000.0 was preceded by a dip in the X-ray flux. The statistical significance of each dip (and the less than significant level of other events that appear to the eye as possible dips) was verified using a probability formula derived by Bernoulli for assessing the likelihood of obtaining $N$ consecutive events that lie more than $1 \sigma$ below the mean. The time delay of about 4 weeks between the X-ray and radio events provides us with an estimate of how far from the black hole the radio jet starts (i.e., the location of the "core"). The answer in $3 \mathrm{C} 120$ is about $0.4 \mathrm{pc}$ after taking into account the

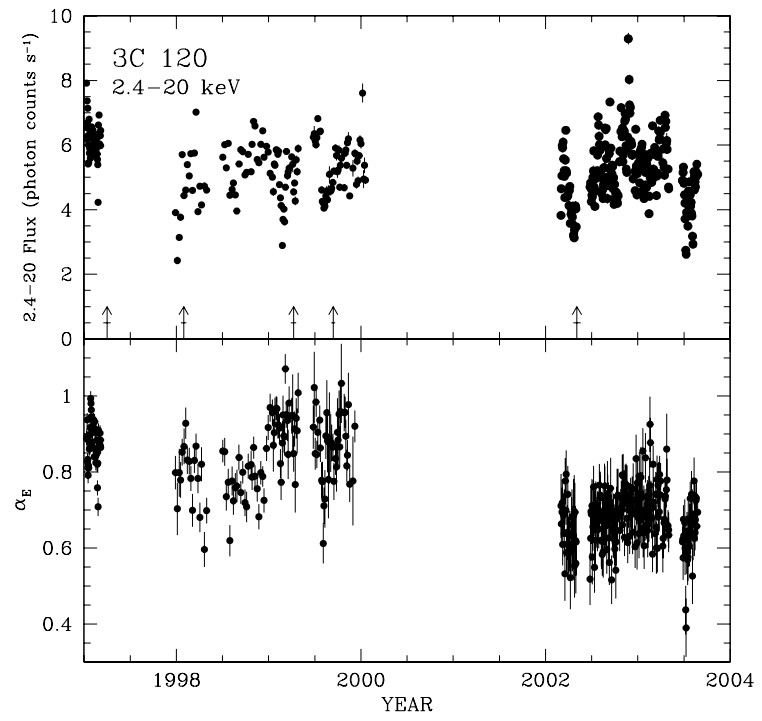

FIGURE 7. Top: X-ray light curve of 3C 120. The extrapolated times when the positions of superluminal radio knots coincided with the core are marked by vertical arrows, with horizontal bars giving the uncertainty in the time of coincidence. Any superluminal features appearing after mid-2003 are not yet apparent in the VLBA data. An outburst at $37 \mathrm{GHz}$ in early September 2003 has been communicated privately by $\mathrm{H}$. Teräsranta; such outbursts have previously corresponded with superluminal ejections. Bottom: X-ray spectral index as a function of time. Note the tendency for the spectrum to flatten at lower flux levels, as is true for black-hole binary systems.

quasi-relativistic effects (mostly light-travel time). The delay also confirms that much of the X-ray emission comes from near the black hole rather than from the radio emitting part of the jet. On the other hand, the low "equivalent width" of the dips compared to the major drop-off seen in microquasars suggests that the jet might contribute as much as a few tens of percent of the X-rays.

We resumed the RXTE monitoring program in 2002. As is seen in Fig. 7, an X-ray dip was again followed by the emergence of a superluminal radio knot from the core in early 2002. A second X-ray dip in mid-2003 was followed by a radio outburst, so we expect to find another superluminal ejection near that time when we collect enough VLBA images to follow changes in the jet.

In March 2004, we will start to monitor a similar radio galaxy, 3C 111, with RXTE and the VLBA in order to determine whether it, too, follows the X-ray dip/superluminal ejection pattern. If it does, then we hope that RXTE will live long enough to determine the mass of 3C 111 in the same manner as has been done for 3C 120 (see Marshall et al., these proceedings). If this is possible, then a dependence on black-hole mass of the 


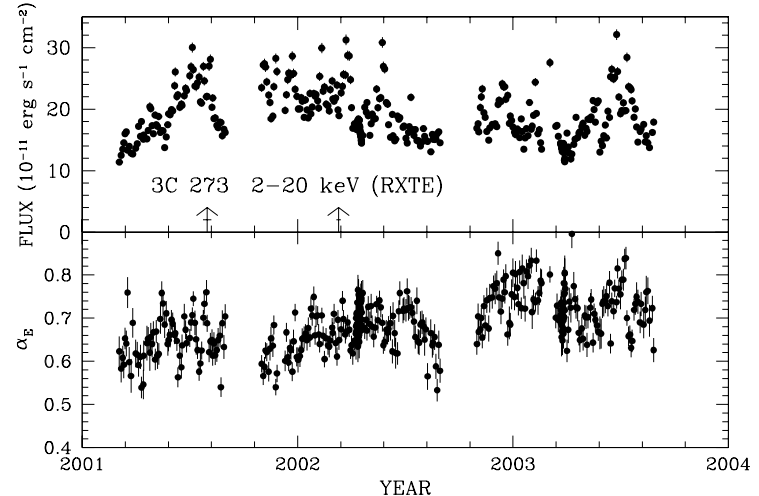

FIGURE 8. RXTE light curve and spectral index of 3C 273 since March 2001.

distance of the radio core from the black hole will be possible. The big question is whether the scaling of jets varies directly with, or as the square-root of, the black hole mass, or something in between.

\section{THE QUASAR 3C 273}

The quasar 3C 273 is another hybrid object: a blazar in the radio and a "normal" quasar in the optical. The source of the strong X-ray flux is controversial, since substantial jet emission is expected, yet the optical characteristics suggest that the accretion disk coronal emission might be prominent as well. In previous RXTE campaigns [2], Xray variations were well correlated with the near-infrared $\mathrm{K}$-band, with the X-rays lagging by $\sim 1$ day. The spectral index in the X-ray is $0.7 \pm 0.1$ (same as for the quiescent IR emission), which is consistent both with inverse Compton emission in the jet and with an origin in the accretion disk/corona.

Our thrice-weekly RXTE monitoring starting in March 2001 (Fig. 8) represents the first systematic long-term monitoring campaign for 3C 273, the brightest X-ray quasar. Note the superposition of short-term (days) flares on more prolonged outbursts.

Kataoka et al. [13] have analyzed archival RXTE data for 3C 273, including the McHardy et al. campaigns in 1997 and 1999. From these irregularly sampled data, Kataoka et al. find a break in the power spectral density (PSD) at $4.7 \pm 1.5 \times 10^{-6} \mathrm{~Hz}$, with the slope changing from -1.4 to -2.4 . The PSD from our more ex-



FIGURE 9. Power spectral density (PSD) $\left(\mathrm{rms}^{2} / \mathrm{Hz}\right.$ vs. frequency in $\mathrm{Hz}$ ) of 3C 273 from RXTE and EXOSAT observations. The smooth curve gives the best-fit model, with parameters fit probability $P$, break frequency $v_{\mathrm{B}}$, low- $v$ slope $\alpha_{\mathrm{L}}$, and high- $v$ slope $\alpha_{\mathrm{H}}$ listed on top. The jagged line represents the data, distorted by Poisson noise and aliasing at high $v$.

tensive data (Fig. 9), in which we've taken into full account the effects of the sampling pattern, shows a similar break, but at $6.4 \times 10^{-7} \mathrm{~Hz}$, with a change in slope also from -1.0 to -2.4 . This is quite similar to the PSD found in black-hole binaries, in which the slope changes from -1.0 to -2.5 at a frequency related to the size of the inner accretion disk and thus to the mass of the black hole $[14,15,16]$. If we scale Cyg X-1 [17, 18] to $3 \mathrm{C} 273$, the ratio of the break frequencies (from PSD fits by McHardy) is $M(3 \mathrm{C} 273) / M(\mathrm{CygX}-1) \sim(6.4 \times$ $\left.10^{-7} \mathrm{~Hz} / 22 \mathrm{~Hz}\right)^{-1}=3 \times 10^{7}$. If we take the mass of the BH in Cyg X-1 to be $10-20 M_{\odot}$, we get $M(3 \mathrm{C} 273) \sim$ $3-6 \times 10^{8} M_{\odot}$, which is, to within the uncertainty, the same as obtained by the reverberation mapping technique [19].

\section{CONCLUSIONS}

Prior to the high-energy/optical-IR/radio timing studies, most blazar scientists operated under the working assumption that higher frequency emission occurs closer to the central engine than the position of the parsec-scale high-frequency radio core. The implication of the results presented above is that the most intense, variable, nonthermal phenomena take place in the radio-emitting portion of the jet, which is imaged by the VLBA at short wavelengths. But the timescales of variability are often less than about one day. Such rapid fluctuations require dimensions smaller than a few tens of light-days even for cases of strong relativistic beaming. This is reconciled by noting that the jets with the highest Lorentz factors, 
greater than about 20 , must be intrinsically very narrow, with half-opening angles less than about $0.5^{\circ}$; otherwise, they would all appear fat given the small angle between the jet axis and the line of sight. Such a narrow conical jet has a transverse radius of only about 10 light-days or less at a distance of $1 \mathrm{pc}$ from its apex. If the thickness of the emission region is similar to its radius, variations on timescales less than a day are possible for objects with Lorentz factors greater than about 10 at such a location. The timescale of variability can therefore be very misleading as an indicator of the site of emission.

As pointed out by Lähteenmäki \& Valtaoja [20], placing the site of the high-energy emission so far from the central engine poses a problem for models in which the $\gamma$-rays result from upscattering of broad emission-line photons by electrons in the jet (e.g., [21]). Since the broad-line region (BLR) is thought to be within $1 \mathrm{pc}$ of the central engine, the angle of scattering is not as favorable as assumed in the models. Reverberation mapping should be carried out on some blazars to measure the size of the BLR in order to check this.

One of the items on the agenda for the future is to confirm the reverse-lag tendency in more objects. This will be possible when the wide-field, highly sensitive GLAST $\gamma$-ray observatory is deployed in a few years. Detailed $\gamma$-ray light curves will finally be available for many objects. This instrument, coupled with the VLBA will allow us to map the variable emission from radio frequencies to high energies armed with the knowledge of where the radiative action is taking place in the jet. It would be great if we could add the X-ray emission to these studies by launching an "emergency" X-ray timing mission before the GLAST era ends!

\section{ACKNOWLEDGMENTS}

This work was supported in part by NASA grants NAG511811 and NAG5-13074 and by National Science Foundation grant AST-0098579.

\section{REFERENCES}

1. Marscher, A. P., and Gear, W. K. Astrophys. J., 298, 114-127 (1985).

2. McHardy, I., et al., Mon. Not. Royal Astron. Soc., 310, 571-576 (1999).

3. Jorstad, S. G., et al., Astrophys. J., 556, 738-748 (2001).

4. Jorstad, S. G., et al., Astrophys. J. Suppl., 134, 181-240 (2001).

5. Vlahakis, N., and Königl, A., Astrophys. J., submitted (also Astro-ph/0310747) (2004).

6. Böttcher, M., et al., Astrophys. J., 596, 847-859 (2003).

7. Stirling, A. M., et al., Mon. Not. Royal Astron. Soc., 341, 405-422 (2003).
8. Villata, M., et al., Astron. Astrophys., 390, 407-421 (2002).

9. Gómez, J. L., et al., Science, 289, 2317-2320 (2000).

10. Grandi, P., et al., Astrophys. J., 487, 636-643 (1997).

11. Mirabel, I. F., and Rodrígez, L. F., Nature, 392, 673-676 (1998).

12. Marscher, A. P., et al., Nature, 417, 625-627 (2002).

13. Kataoka, J., et al. Mon. Not. Royal Astron. Soc., 336, 932-944 (2002).

14. McHardy, I. M., Papadakis, I. E., and Uttley, P. Nuclear Phys. B, 69, 509 (1998).

15. Edelson, R., and Nandra, K. Astrophys. J., 514, 682-690 (1999).

16. Markowitz, A., et al., Astrophys. J., 593, 96-114 (2003).

17. Cui, W., et al., Astrophys. J. Lett., 474, L57-L60 (1997).

18. Novak, M. A., Vaughan, B. A., Wilms, J., Dove, J. B., and Begelman, M. C. Astrophys. J., 510, 874-891 (1999).

19. Kaspi, S., et al., Astrophys. J., 533, 631-649 (2000).

20. Lähteenmäki, A., and Valtaoja, E., Astrophys. J., 590, 95-108 (2003).

21. Sikora, M., Begelman, M. C., and Rees, M. J. Astrophys. J., 421, 153-162 (1994). 